\title{
Effect of temperature, relative humidity and rainfall on dengue fever and leptospirosis infections in Manila, the Philippines
}

\author{
A. SUMI ${ }^{1 *}$, E. F. O. TELAN ${ }^{2}$, H. CHAGAN-YASUTAN ${ }^{3}$, M. B. PIOLO ${ }^{4}$, \\ T. HATTORI ${ }^{5}$ AND N. KOBAYASHI ${ }^{1}$ \\ ${ }^{1}$ Department of Hygiene, Sapporo Medical University School of Medicine, Sapporo, Hokkaido, Japan \\ ${ }^{2}$ National Reference Laboratory for HIVIAIDS, Hepatitis, and other STDs, STD/AIDS Cooperative Central \\ Laboratory, Manila, Philippines \\ ${ }^{3}$ Division of Disaster-Related Infectious Disease, International Research Institute of Disaster Science, Tohoku \\ University, Sendai, Miyagi, Japan \\ ${ }^{4}$ Epidemiology Unit, San Lazaro Hospital, Manila, Philippines \\ ${ }^{5}$ Graduate School of Health Science Studies, Kibi International University, Takahashi, Okayama, Japan
}

Received 18 March 2016; Final revision 24 July 2016; Accepted 12 August 2016;

first published online 9 September 2016

\section{SUMMARY}

Dengue fever (DF) and leptospirosis are serious public problems in tropical regions, especially in Manila, the Philippines. In attempting to understand the causes of DF and leptospirosis seasonality, meteorological factors have been suspected, but quantitative correlation between seasonality and meteorological factors has not been fully investigated. In this study, we investigated correlation of temporal patterns of reported numbers of laboratory-confirmed cases of both DF and leptospirosis with meteorological conditions (temperature, relative humidity, rainfall) in Manila. We used time-series analysis combined with spectral analysis and the least squares method. A 1-year cycle explained underlying variations of DF, leptospirosis and meteorological data. There was a peak of the 1-year cycle in temperature during May, followed by maxima in rainfall, relative humidity and number of laboratory-confirmed DF and leptospirosis cases. This result suggests that DF and leptospirosis epidemics are correlated not only with rainfall but also relative humidity and temperature in the Philippines. Quantifying the correlation of DF and leptospirosis infections with meteorological conditions may prove useful in predicting DF and leptospirosis epidemics, and health services should plan accordingly.

Key words: Dengue fever, disaster, leptospirosis, time-series analysis, weather conditions.

\section{INTRODUCTION}

Neglected tropical diseases (NTDs) are infections that primarily occur in regions of rural poverty in developing countries and affect more than one billion people, costing developing economies billions of dollars every year [1]. However, some of the NTDs also occur in

\footnotetext{
* Author for correspondence: Dr A. Sumi, Department of Hygiene, Sapporo Medical University School of Medicine, S-1, W-17, Chuo-ku, Sapporo 060-8556, Japan.

(Email: sumi@sapmed.ac.jp)
}

urban slums and favelas [2]. The NTDs dengue fever (DF), leptospirosis and rabies are a particular cause of urban health problems in developing countries. For both DF and leptospirosis, urban flooding, at times of natural disaster, is a key component of transmission. As global warming results in increased flooding in some regions of the developing world, the combined effects of urbanization and climate change may lead to an increased incidence of these two infections in the coming decades [2]. As a result of climate change, there is also a possibility that these NTDs 
could emerge in urban regions of the United States [3]. Therefore, DF and leptospirosis are important public health issues worldwide.

Several studies have shown that leptospirosis is often misdiagnosed as DF and underdiagnosed in endemic regions [4]. DF is transmitted by mosquitoes and leptospirosis is a zoonotic infectious disease caused by Leptospira bacteria, which can be found in fresh water contaminated by animal urine. Epidemics of DF and leptospirosis have been observed following natural disasters [5] and some of the most affected nations are disaster-prone island countries [6]. In the Republic of the Philippines, DF and leptospirosis are recognized as common disaster-related infections, in addition to diarrhoea and cholera [7].

A clear relationship has been observed between an increase in DF cases and the southwest and northeast monsoons, particularly in Manila, in the Philippines [8-10]. Moreover, leptospirosis is associated with floodwaters following monsoons [11-13]. It has also been suggested that temperature and rainfall considerably increase the incidence of DF infections [14, 15]. One study that assessed climatic factors associated with DF incidence in Manila showed that heavy rainfall in the wet season from June to December was significantly associated with increased DF incidence over 1996-2005 [15]. Leptospirosis outbreaks also typically occur during the typhoon season (July-October) [16].

Recently, the World Health Organization performed a case study to investigate the correlation between DF and leptospirosis incidence with meteorological factors such as rainfall, temperature and relative humidity in Sri Lanka, with the aim of developing models to predict the effects of climatic variations on disease incidence [14]. Time-series analysis revealed an association between disease epidemics and meteorological factors, which was not visually apparent. DF and leptospirosis are also serious public health problems in Manila; however, to date, time-series analysis has not been employed to examine the effect of meteorological variables on disease occurrence in the Philippines.

The present research is designed to shed light on the relationship between climate factors of rainfall, temperature and relative humidity on the occurrence of DF and leptospirosis in Manila over the period January 2013December 2014, which encompasses Typhoon Haiyan (known in the Philippines as Typhoon Yolanda). This was one of the strongest tropical cyclones ever recorded, devastating portions of Southeast Asia, especially the Philippines, in early November 2013. It is hoped that the work will provide insight into the relationship between meteorological factors and the incidence of DF and leptospirosis [14]. The findings could also inform prevention and control of the diseases.

\section{DATA}

\section{Study area}

The Philippines comprises 7107 islands in Southeast Asia, with the three island groups of Luzon, Visayas, and Mindanao split into 17 regions [1]. The population is 92337852 (2010 census), with an average annual population growth rate of $1.90 \%$ over the period 2000-2010. A large proportion of the population $(37 \cdot 3 \%)$ lives in three regions, namely Calabarzon (16.03 million people), Manila (11.55 million people), and Central Luzon (9.72 million people) [17]. The country has a tropical marine climate, with an annual dry season from December to May and annual wet season from June to November [17]. Typhoons usually occur from June to September. We conducted the present study at San Lazaro Hospital in Manila, a national 500-bed referral infectious disease centre for Manila and neighbouring provinces, which mostly serves economically disadvantaged people and is one of the sentinel hospitals of the Philippines Integrated Disease Surveillance and Response (PIDSR) system.

\section{DF and leptospirosis data}

We used the weekly number of cases of laboratoryconfirmed DF and leptospirosis from January 2013 to December 2014, collected over 104 weeks (104 data points) at San Lazaro Hospital via the PIDSR system.

Laboratory confirmations for DF were conducted under strategies of the PIDSR system as follows: (i) isolation of the dengue virus from serum, plasma or leukocytes; (ii) demonstration of $\geqslant$ fourfold change in reciprocal $\mathrm{IgG}$ or $\mathrm{IgM}$ antibody titres to one or more dengue virus antigens in paired serum samples; (iii) detection of viral genomic sequences in serum or cerebrospinal fluid samples by polymerase chain reaction (PCR) [18]. Laboratory confirmations of leptospirosis were conducted under strategies of the PIDSR system as follows: (i) isolation (and typing) from blood or other clinical materials through cultures of pathogenic Leptospira; (ii) positive serology, preferably microscopic agglutination testing (MAT) or PCR, using a range of Leptospira strains for antigens that should be representative of local strains [18]. 
Table 1. Summary statistics of weekly meteorological conditions in Manila, the Philippines

\begin{tabular}{llllll}
\hline \hline Variable & Minimum & Median & Mean & Maximum & s.D. \\
\hline $\begin{array}{c}\text { Mean temp. } \\
\left({ }^{\circ} \mathrm{C}\right)\end{array}$ & $24 \cdot 6$ & $28 \cdot 3$ & 28.4 & $31 \cdot 5$ & $1 \cdot 4$ \\
$\begin{array}{c}\text { Relative } \\
\text { humidity }\end{array}$ & $62 \cdot 3$ & $73 \cdot 5$ & $74 \cdot 4$ & $89 \cdot 9$ & $6 \cdot 5$ \\
$\begin{array}{c}\text { Rainfall } \\
(\mathrm{mm})\end{array}$ & $0 \cdot 0$ & $16 \cdot 4$ & $48 \cdot 2$ & 632.5 & $83 \cdot 0$ \\
\hline \hline
\end{tabular}

\section{Meteorological data}

Data on daily maximum and minimum temperatures, relative humidity, and rainfall for Manila were collected by the Philippine Atmospheric, Geophysical, and Astronomical Service Administration in Quezon City during 2013 and 2014. The daily data were gathered over a total of 730 days during the aforementioned period (730 data points). Daily average temperatures were computed as means of daily maxima and minima. Weekly means of average temperature, relative humidity and total rainfall were calculated from daily records.

Descriptive statistics for the weekly meteorological data are shown in Table 1 . Mean weekly average values in Manila were: mean temperature $28.4{ }^{\circ} \mathrm{C}$, relative humidity $74 \cdot 4 \%$, and total rainfall $48 \cdot 2 \mathrm{~mm}$.

\section{METHODS}

We used time-series analysis combined with spectral analysis based on the maximum entropy method (MEM) in the frequency domain and least squares method (LSM) in the time domain, as proposed in our previous work $[19,20]$. For explanation of the time-series analysis, we used time-series data of the reported case of DF shown in Figure $1 a$.

\section{Spectral analysis}

We assumed that the original time-series data $x(t)$ $(t=$ time) in Figure $1 a$ were composed of systematic and fluctuating parts [21]:

$x(t)=$ systematic part + fluctuating part.

To investigate temporal patterns of $x(t)$, we performed MEM spectral analysis, which is useful for investigating periodicities of short time-series as used in the present study [22]. That analysis produces a power spectral density (PSD), from which we obtain the power representing the amount of amplitude of $x(t)$ at each frequency (note the reciprocal relationship between the scales of frequency and period). The formulation of MEM-PSD has been described previously [20]. Figure $2 a$ shows the PSD for the time-series data in Figure $1 a$.

\section{LSM}

The validity of the MEM spectral analysis results was confirmed by calculation of the least squares fitting (LSF) curve to the original data $x(t)$ (Fig. 1a) with MEM-estimated periods. The formulation of the LSF curve in $X(t)$ is described as follows [20]:

$X(t)=A_{0}+\sum_{n=1}^{N_{p}} A_{n} \cos \left\{2 \pi f_{n}\left(t+\theta_{n}\right)\right\}$,

which is calculated using the LSM for $x(t)$ (Fig. 1a) with unknown parameters $f_{n}, A_{0}$ and $A_{n}(n=1,2$, $\left.3, \ldots, N_{p}\right)$, where $f_{n}\left(=1 / T_{n}: T_{n}\right.$ is the period) is the frequency of the $n$th component, $A_{0}$ is a constant that indicates the average value of the time-series data, $A_{n}$ and $\theta_{n}$ are the amplitude and phase of the $n$th component, respectively, and $N_{p}$ is the total number of components.

Figure $1 a$ shows the LSF curve calculated using three MEM-estimated periods observed in Figure $2 a$. Reproducibility of the modified time-series data using the optimum LSF curve was evaluated by the Pearson correlation coefficient $(\rho)$ with SPSS software v. 17.0J for Windows (SPSS Inc., USA). To investigate seasonality of the time-series data, their LSF curve was determined using a seasonal cycle corresponding to a 1-year period $T_{1}$ (Fig. $3 a$ ).

\section{RESULTS}

\section{Temporal variations of DF, leptospirosis and meteorological data}

The weekly time-series data used are depicted in Figure 1. The seasonal pattern of DF data (Fig. 1a) indicates large values during the wet season (JuneNovember) with a peak in September, contemporaneous with high temperature, high relative humidity and heavy rainfall (Fig. 1c-e). A large peak in the number of leptospirosis cases (Fig. 1b) was observed in September 2013, and a small peak in September 2014. The temporal pattern of temperature (Fig. 1c) reveals an increase in the dry season (DecemberMay), with peaks in April 2013 and May 2014. Thereafter, the temperature remained around $28^{\circ} \mathrm{C}$ 

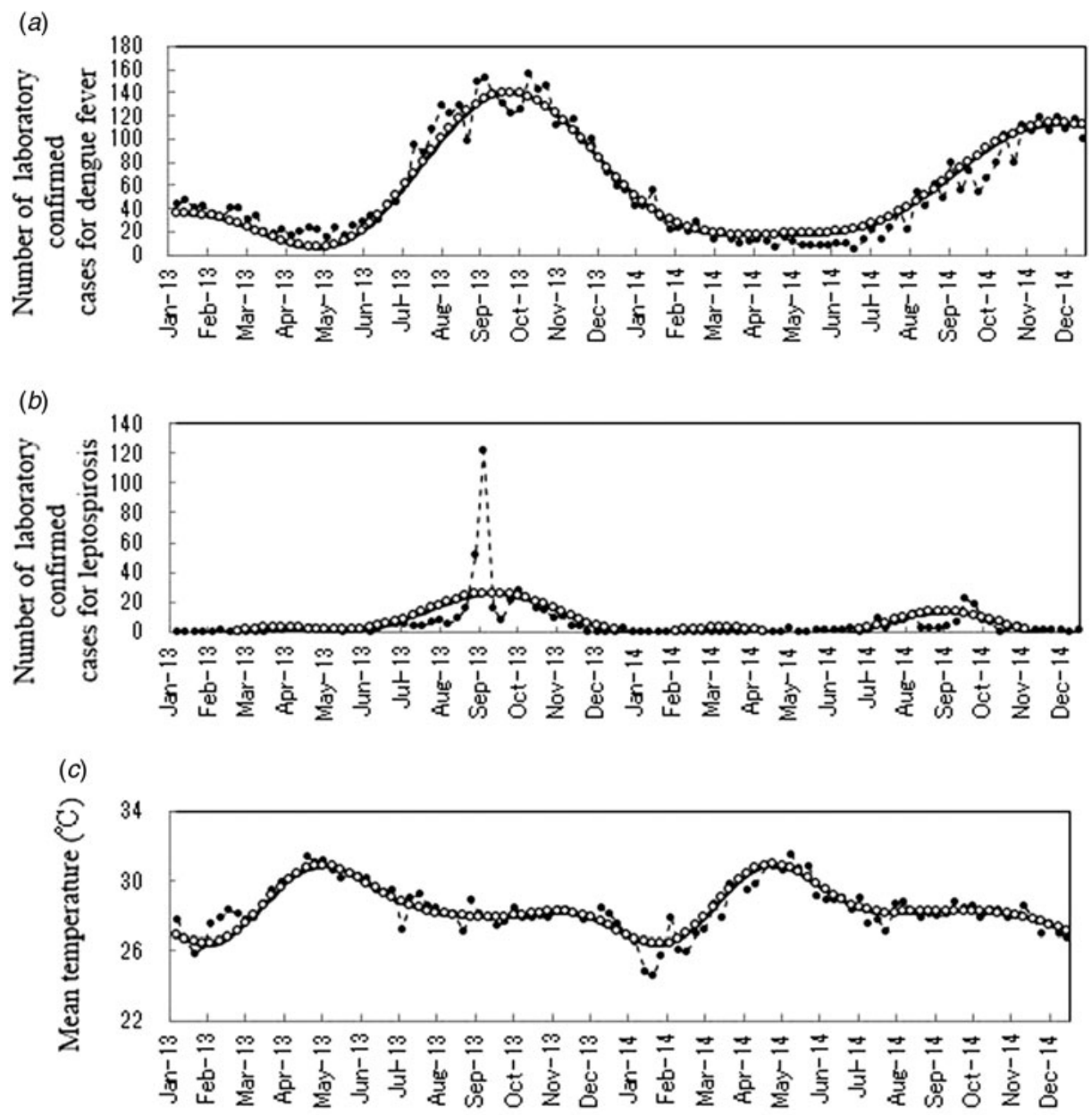

(d)
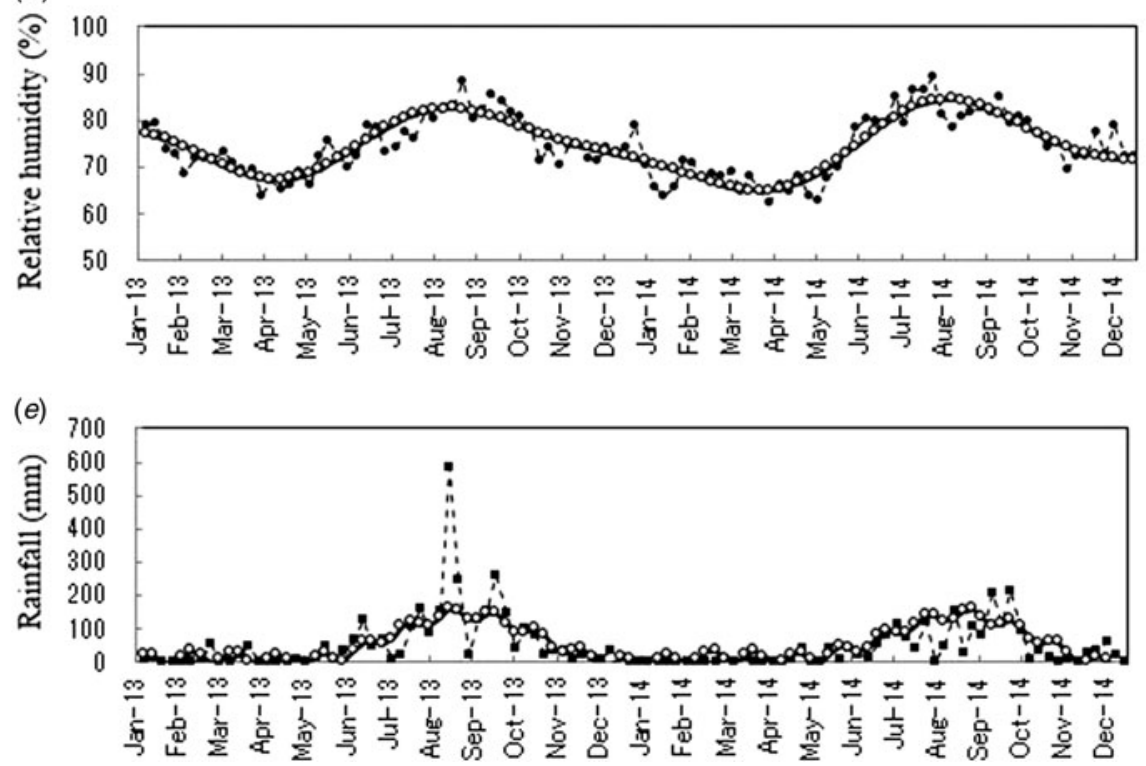

Fig. 1. Comparison of least-squares-fitting curve calculated for three dominant periods (-O-) with original data (- -0- -), for: $(a)$ dengue fever, $(b)$ leptospirosis, $(c)$ mean temperature, $(d)$ relative humidity, $(e)$ rainfall. 

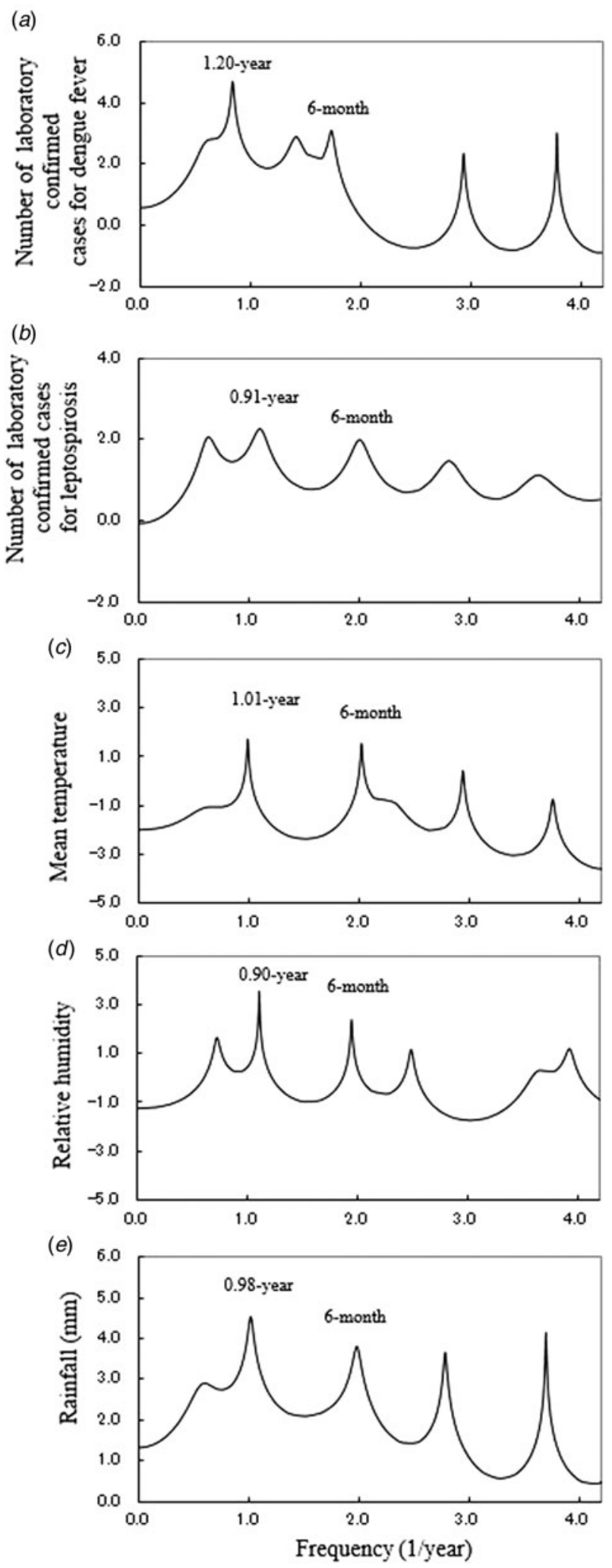

Fig. 2. Power spectral density of the original data for: $(a)$ dengue fever, $(b)$ leptospirosis, $(c)$ mean temperature, $(d)$ relative humidity, $(e)$ rainfall. in the wet season (June-November). The seasonal pattern of relative humidity (Fig. 1d) indicates large values during the wet season, with peaks in August 2013 and July 2014. The seasonal pattern of rainfall (Fig. 1e) shows maxima during the wet season, with a peak in August 2013. There was a smaller maximum during the 2014 wet season, with a peak in October.

\section{Spectral and LSF analyses}

PSDs for the original time-series data in Figure 1 are shown in Figure 2. In each PSD, the most prominent spectral line was at frequency $f=1 \cdot 0$ [units (1/year)], corresponding to the 1-year cycle, that is, the seasonal cycle: 1.20 year, 0.91 year, 1.01 year, 0.90 year and 0.98 year for DF (Fig. 2a), leptospirosis (Fig. 2b), temperature (Fig. 2c), relative humidity (Fig. 2d) and rainfall (Fig. 2e), respectively. In Figure $2 a$, a spectral peak in the low-frequency range of PSD $(f<1 \cdot 0)$ reflects oscillations longer than the 1-year cycle.

Three dominant spectral-peak frequency modes are shown in Table 2, with corresponding periods and intensities (powers) of the peaks. In Figure 1, each LSF curve calculated with three dominant periods reproduces the modified data well. Thus, the periods detected by MEM spectral analysis for each original datum (Fig. 2, Table 2) were confirmed to be accurate. The good fit of each LSF curve to the original data was supported by the fact that the $\rho$ values between the modified data and the LSF curve were large. They were 0.96 , $0 \cdot 60,0 \cdot 90,0 \cdot 88$ and $0 \cdot 67$ for DF, leptospirosis, temperature, relative humidity and rainfall data, respectively.

\section{Seasonal cycle of DF and leptospirosis data and meteorological data}

The LSF curves calculated with the seasonal cycles for DF (1.20-year) and leptospirosis (0.91-year) were normalized in amplitude, and are overlaid in Figure 3 ( $a, b$, respectively). In Figure 3, oscillation is shown in coordinate phase between the LSF curve for numbers of laboratory-confirmed DF and leptospirosis cases and the LSF curve for meteorological conditions (temperature, rainfall, relative humidity). Peaks of the LSF curves for relative humidity, rainfall and temperature data precede those for DF data by 1, 2 and 4 months, respectively, and those for leptospirosis data by 0,1 and 3 months. The LSF curve for DF (Fig. $3 a$ ) showed significant correlations with that for 

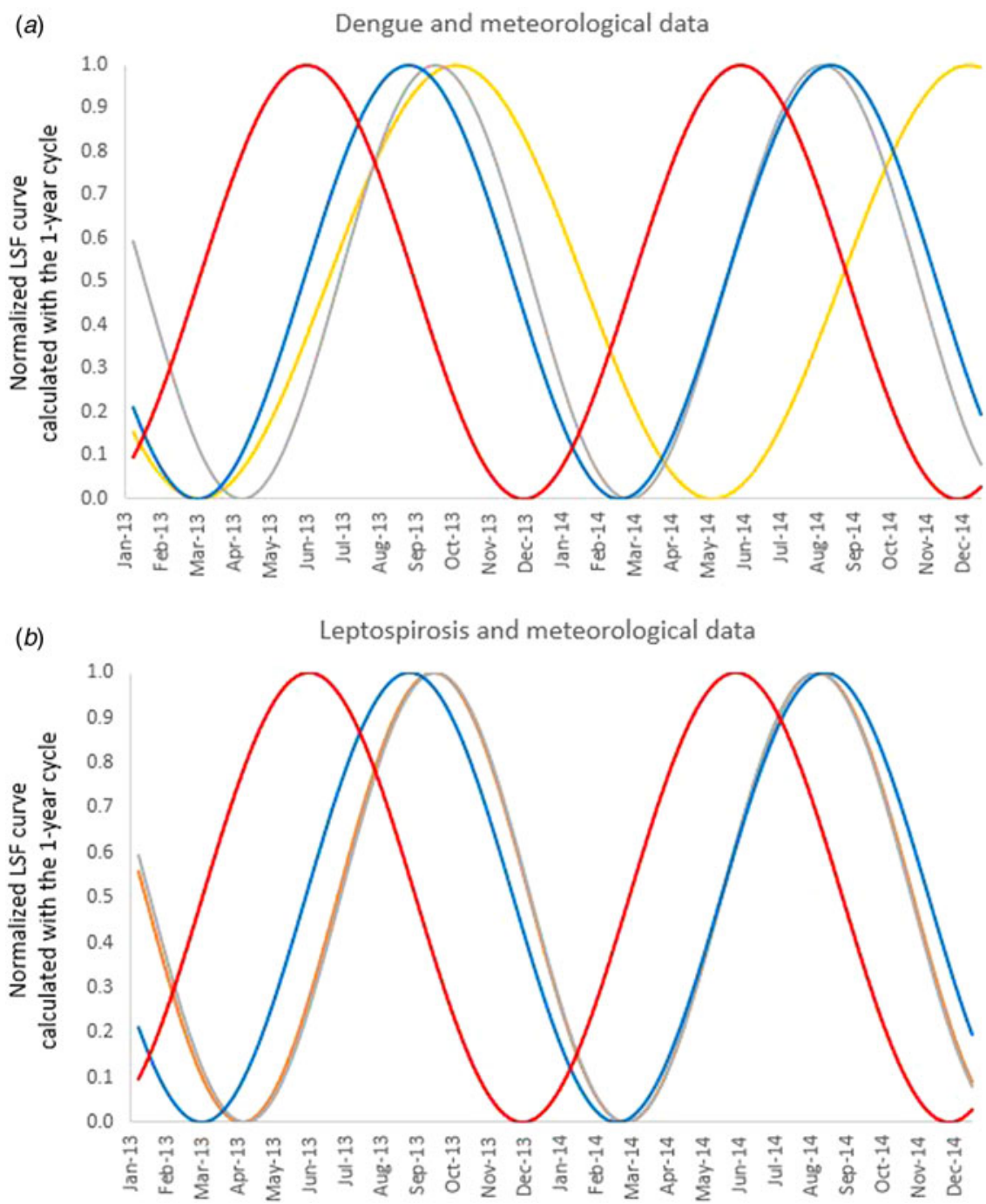

Fig. 3. Normalized least-squares-fitting (LSF) curves calculated with the seasonal cycle, for: (a) dengue fever (orange), mean temperature (red), rainfall (blue) and relative humidity (grey); (b) leptospirosis (orange), mean temperature (red), rainfall (blue) and relative humidity (grey). In panel (b), LSF curves of leptospirosis (orange) and relative humidity (grey) overlap.

meteorological data (Table 3), i.e. $-0 \cdot 60,0 \cdot 45$ and $0 \cdot 51$ for mean temperature, relative humidity and rainfall, respectively $(P<0 \cdot 01)$. For leptospirosis, the LSF curve (Fig. 3b) showed significant correlations with the LSF curves for mean temperature and rainfall (0.99 and 0.92 , respectively, $P<0.01)$ but no significant correlation with mean temperature $(-0 \cdot 03, P=0 \cdot 74)$.

\section{DISCUSSION}

There was a peak in temperature during May, followed by maxima in rainfall, relative humidity and number of DF patients (Fig. 3). We assumed that this result shows a relationship between monsoons and DF occurrence in the Philippines. In summer, temperature rises, rainfall increases with moist air transport from the ocean to continent, a monsoon begins and the atmosphere moistens, increasing relative humidity. This was followed by a peak in the number of DF patients. An earlier study by Su [8] addressed the reasons for DF epidemics in terms of rainfall. We assumed that DF epidemics are correlated not only with rainfall but also relative humidity and temperature. DF during a week was related to rainfall over the prior 6-7 weeks. This can be attributed to the life-cycle duration of mosquitoes and the requirement of an adequate number of cases for spread, which is in turn affected by population density [15].

The disease leptospirosis is usually transmitted to humans through wild rats and their urine. However, 
Table 2. Characteristics of the three dominant spectral peaks shown in Figure 2

\begin{tabular}{llll}
\hline \hline Variable & $f(1 /$ year $)$ & Period (year) & Power \\
\hline Weekly no. of laboratory-confirmed cases for dengue fever & $0 \cdot 84$ & $1 \cdot 20$ & $1646 \cdot 20$ \\
& 1041 & $0 \cdot 71$ & $128 \cdot 41$ \\
& $1 \cdot 73$ & $0 \cdot 58$ & $76 \cdot 85$ \\
Weekly no. of laboratory-confirmed cases for leptospirosis & $0 \cdot 63$ & $1 \cdot 58$ & $18 \cdot 98$ \\
& $1 \cdot 10$ & $0 \cdot 91$ & $33 \cdot 35$ \\
Mean temperature & $2 \cdot 01$ & $0 \cdot 50$ & $19 \cdot 84$ \\
& $0 \cdot 99$ & $1 \cdot 01$ & $0 \cdot 84$ \\
Relative humidity & $2 \cdot 02$ & $0 \cdot 49$ & $0 \cdot 71$ \\
& $2 \cdot 95$ & $0 \cdot 34$ & $2 \cdot 94$ \\
Rainfall & $0 \cdot 73$ & $1 \cdot 38$ & $25 \cdot 33$ \\
& $1 \cdot 11$ & $0 \cdot 90$ & $3 \cdot 95$ \\
& $1 \cdot 95$ & $0 \cdot 51$ & $2043 \cdot 00$ \\
\hline \hline
\end{tabular}

Table 3. Spearman's $\rho$ calculated for original data and least-squares-fitting curves with seasonal cycles

\begin{tabular}{llll}
\hline \hline & Mean temp. & Relative humidity & Rainfall \\
\hline Dengue fever & $-0 \cdot 60^{* *}$ & $0 \cdot 45^{* *}$ & $0 \cdot 51^{* *}$ \\
Leptospirosis & -0.03 & $0 \cdot 99^{* *}$ & $0 \cdot 92^{* *}$ \\
\hline \hline
\end{tabular}

$* * P<0 \cdot 01$.

bacteria in floodwater can also infect humans by entering the body through cuts and skin abrasions. Libraty et al. [13] reported that the maximum occurrence of leptospirosis was in October, 2-3 months later than the peak occurrence of dengue in Thailand. However, in the present study of Manila, the peak occurrence of leptospirosis preceded that of DF by only 1 month (Fig. $3 a, b$ ). This difference in peak month of leptospirosis cases between Thailand and Manila may be caused by epidemiological characteristics of this disease in each country. In Thailand, leptospirosis outbreaks from cultivation of rice and other agriculture have become a problem [13]. In Manila, large-scale leptospirosis epidemics have occurred after heavy rains and flooding following monsoons [16]. It should also be noted that both of these diseases are caused after natural disasters, which are increasing year by year [23]. Differential diagnoses of these febrile illnesses is an important issue, and the development of point-of-care testing and metrological analysis described herein would aid such diagnoses [24].

Floods, and a fortiori urban floods, have often induced outbreaks of hantavirus infection, i.e. haemorrhagic fever with renal syndrome (HFRS), whose symptoms can almost completely overlap those of leptospirosis [25]. However, HFRS, which has been limited to Eurasia [26], has not been targeted in the PIDSR system. This is partly because there have been no documented cases of HFRS in Filipino patients [27]. Like HFRS, hantavirus pulmonary syndrome (HPS) has not been considered in the PIDSR system, because HPS has been limited to the Americas [26]. However, evidence is now mounting for clinical and pathological overlap between HPS and HFRS [28, 29]. Consequently, hantavirus infections should be considered a cause of acute respiratory distress in all endemic areas worldwide [28]. Indeed, Quelapio et al. [27] provided substantial evidence that hantavirus infection does occur in the Philippines. New hantaviruses, different hosts and human syndromes are expected to be discovered, especially in Southeast Asia. There, murid rodents and Soricomorpha small mammals [30] are endemic and highly diversified, and human populations are regularly exposed by contact, as in the Philippines [31, 32]. Increased awareness of hantavirus infection in the PIDSR system would make it efficient to estimate the burden of such infection and prevent it in the Philippines.

In general, meteorological phenomena are nonstationary and nonlinear, and transit from one state to another in a complicated manner. It is possible that the correlation strengths of seasonal cycles for DF and leptospirosis data with those of meteorological data (Fig. 3, Table 3) also change temporally. To elucidate the temporal evolution of such nonlinear phenomena, it is preferable to deal with shorter time-series 
through segment-based time-series analysis [22]. For example, this was done in our previous studies on measles using a 56-year data duration [33] and influenza using a 51-year duration [34]. The longer duration data ( $>2$ years) on DF and leptospirosis in the present study (Fig. $1 a, b$, respectively) enabled further investigation of temporal variations for correlations of DF and leptospirosis infections with meteorological conditions, using segment-based timeseries analysis. Further accumulation of DF and leptospirosis data in the PIDSR system will contribute to future investigations of meteorological factors for DF and leptospirosis transmission in the Philippines.

The present results suggest that DF and leptospirosis cases in Manila were influenced by monsoon occurrence. Further time-series analyses of the two diseases and meteorological data in other regions of the world may elucidate their potential relationships to the monsoon or other epidemiological factors.

\section{ACKNOWLEDGEMENTS}

This study was supported by the Philippines Atmospheric Geophysical and Astronomical Services Administration (PAGASA), a special research grant from the International Research Institute of Disaster Science (IRIDeS) of Tohoku University, and JSPS KAKENHI grant nos. JP16K09061, JP26257506, JP25460769, JP25305022. The first author also thanks the students of Sapporo Medical University School of Medicine (R. Inoue, M. Senda, Y. Teguri, K. Nakajima and M. Yashiro) for their help with discussion of the topic.

\section{DECLARATION OF INTEREST}

None.

\section{REFERENCES}

1. WHO. Neglected tropical diseases (http://www.who.int/ neglected_diseases/en/). World Health Organization.

2. Hotez PJ. Forgotten People, Forgotten Diseases: The Neglected Tropical Diseases and their Impact on Global Health and Development, 2nd edn. Herndon: ASP Press, 2010.

3. Gubler DS, et al. Climate variability and change in the United States: potential impacts on vector- and rodentborne diseases. Environmental Health Perspectives 2001; 109 (Suppl. 2): 223-233.

4. Vijayachari P, Sugunan AP, Shriram AN. Leptospirosis: an emerging global public health problem. Journal of Biosciences 2008; 33: 557-569.
5. Watson JT, Gayer M, Connolly MA. Epidemics after natural disasters. Emerging Infectious Diseases 2007; 13: $1-5$.

6. Guha-Sapir D, Hoyois P, Below R. Annual Disaster Statistical Review 2014: the numbers and trends. Université catholique de Louvain, Brussels, Belgium (http://cred.be/sites/default/files/ADSR_2014.pdf).

7. Usuzawa M, et al. Awareness of disaster reduction frameworks and risk perception of natural disaster: a questionnaire survey among Philippine and Indonesian health care personnel and public health students. Tohoku Journal of Experimental Medicine 2014; 233: 43-48.

8. Su GLS. Correlation of climatic factors and dengue incidence in Metro Manila, Philippines. Ambio 2008; 37: 292-294.

9. Buczak AL, et al. Prediction of high incidence of dengue in the Philippines. PLoS Neglected Tropical Diseases 2014; 8: e2771.

10. Hii YL, et al. Forecast of dengue incidence using temperature and rainfall. PLoS Neglected Tropical Diseases 2012; 6: e1908.

11. Easton A. Leptospirosis in Philippine flood. British Medical Journal 1999; 319: 212.

12. Pappas G, et al. The globalization of leptospirosis: worldwide incidence trends. International Journal of Infectious Diseases 2008; 12: 351-357.

13. Libraty DH, et al. A comparative study of leptospirosis and dengue in Thai children. PLoS Neglected Tropical Diseases 2007; 1: e1111.

14. WHO. Climatic factors and the occurrence of dengue fever, dysentery and leptospirosis in Sri Lanka 19962010: a retrospective study (http://www.who.int/kobe centre/publications/climatic_factors_srilanka_2014/en/). World Health Organization.

15. Bravo L, et al. Epidemiology of dengue disease in the Philippines (2000-2011): a systematic literature review. PLoS Neglected Tropical Diseases 2014; 8: e3027.

16. Amilasan AT, et al. Outbreak of leptospirosis after flood, the Philippines, 2009. Emerging Infectious Diseases 2012; 18: 91-94.

17. WHO. Climate change country profiles: Philippines 2012 (http://www.wpro.who.int/environmental_health/ documents/docs/PHL_92EC.pdf). World Health Organization.

18. National Epidemiology Center, Department of Health, Philippines. Manual of Procedures for the Philippine Integrated Disease Surveillance and Response, Vol. 1, 3rd edn, 2014.

19. Sumi A, et al. Time-series analysis of hepatitis A, B, C and $\mathrm{E}$ infections in a large Chinese city: application to prediction analysis. Epidemiology and Infection 2013; 141: 905-915.

20. Harigane $\mathbf{K}$, et al. The role of temperature in reported chickenpox cases from 2000 to 2011 in Japan. Epidemiology and Infection 2015; 143: 2666-2678.

21. Armitage P, Berry G, Matthews JNS. Statistical Method in Medical Research, 4th edn. Oxford: Blackwell Science, 2012.

22. Ohtomo N. et al. New method of time series analysis and its application to Wolf's sunspot number data. 
Japanese Journal of Applied Physics 1994; 33: 23212831.

23. Leaning J, Guha-Sapir D. Natural disasters, armed conflict, and public health. New England Journal of Medicine 2013; 369: 1836-1842.

24. Hattori T, et al. Development of point of care testing for disaster-related infectious diseases. Tohoku Journal of Experimental Medicine 2016; 238: 287-293.

25. Clement J, Maes P, Ranst MV. Acute kidney injury in emerging, non-tropical infections. Acta Clinica Belgica 2007: 62: 387-395.

26. Bi Z, Formenty PBH, Roth CE. Hantavirus infection: a review and global update. Journal of Infection in Developing Countries 2008; 2: 3-23.

27. Quelapio ID, et al. TE. Seroepidemiology of hantavirus in Philippines. International Journal of Infectious Diseases 2000; 4: 104-107.

28. Rasmuson J, et al. Time to revise the paradigm of hantavirus syndromes? Hantavirus pulmonary syndrome caused by European hantavirus. European Journal of Clinical Microbiology \& Infectious Diseases 2011; 30: 685-690.
29. Clement J, Maes P, Ranst MV. Hemorrhagic fever with renal syndrome in the new, and hantavirus pulmonary syndrome in the old world: Paradi(se)gm lost or regained? Virus Research 2014; 187: 55-58.

30. Klein TA, et al. Hantaan virus surveillance targeting small mammals at Nightmare Range, a high elevation military training area, Gyeonggi Province, Republic of Korea. PLoS ONE. Published online: 15 April 2015. doi:10.1371/journal.pone.0118483.

31. Herbreteau V, et al. Is human hantavirosis underestimated in South Asia? Mammal Study 2005; 30: S83-S85.

32. Anyamba A, et al. Developing global climate anomalies suggest potential disease risks for 2006-2007. International Journal of Health Geographics 2006; 5: 60-68.

33. Luo T, et al. Study on the effect of measles control programmed on periodic structures of disease epidemics in a large Chinese city. Epidemiology and Infection 2011; 139: 257-264.

34. Sumi A, et al. Time series analysis of incidence data of influenza in Japan. Journal of Epidemiology 2011; 21: 21-29. 